\title{
Consenso Brasileiro para Transplante de Células-Tronco Hematopoéticas para Tratamento de Doenças Autoimunes
}

\section{Brazilian Consensus on Hematopoietic Stem Cell Transplantation for Autoimmune Diseases}

Júlio C. Voltarelli ${ }^{l}$

Daniela A. Moraes ${ }^{1}$

Andreza A.F. Ribeiro ${ }^{2}$

Maria Carolina de Oliveira ${ }^{1}$

Morgani Rodrigues ${ }^{2}$

Doralina G. Brum ${ }^{l}$

Amilton A. Barreira ${ }^{l}$

Nelson Hamerschlak ${ }^{2}$

\begin{abstract}
Neste trabalho, foram revisadas a literatura internacional e a experiência nacional com transplante de células-tronco hematopoéticas (TCTH) para doenças autoimunes. A evidência acumulada indica que o TCTH autólogo pode beneficiar pacientes com esclerose múltipla em fase inflamatória, refratária aos tratamentos medicamentosos disponíveis, e pacientes com esclerose sistêmica cutânea difusa de caráter progressivo, com ou sem comprometimento sistêmico. Esse tratamento deveria ser disponibilizado na rede pública de saúde, numa fase inicial, em centros de referência com experiência em TCTH e no manejo clínico de doenças autoimunes sistêmicas graves. Rev. Bras. Hematol. Hemoter. 2010;32(Supl. 1):125-135.
\end{abstract}

Palavras-chave: Transplante de células-tronco hematopoéticas; doenças autoimunes; esclerose múltipla; esclerose sistêmica.

\section{Introdução}

Dentre as dezenas de doenças autoimunes graves e refratárias que tiveram remissão induzida com transplante de células-tronco hematopoéticas (TCTH), autólogo ou alogênico, a Reunião de Consenso definiu a esclerose múltipla e a esclerose sistêmica como as únicas doenças, no momento, a merecerem indicação de TCTH em um contexto clínico. A primeira, por acumular uma experiência positiva de aproximadamente quinhentos pacientes transplantados em várias partes do mundo, inclusive no Brasil, como revisado a seguir, e a esclerose sistêmica (ES), pela falta de alternativas terapêuticas eficientes além do TCTH. O nível de evidência para as duas doenças seria I-B (forte recomendação por um grande número de estudos clínicos não randomizados), a qual poderá ser reforçada e se tornar I-A proximamente quando forem publicados os estudos randomizados que estão sendo conduzidos na Europa e nos EUA. ${ }^{1}$
No diabetes mellitus do tipo 1 recém-diagnosticado, apesar dos resultados bastantes favoráveis do estudo clínico brasileiro com TCTH autólogo, ${ }^{2}$ ele deve ser replicado em outros centros, e a real taxa de recaída ser determinada antes de ser adotado clinicamente.

\section{Indicações de TCTH para esclerose sistêmica}

A esclerose sistêmica é uma doença autoimune em que a pele e, frequentemente, órgãos internos são envolvidos. O quadro costuma ter início a partir de hiperreatividade vascular e alterações endoteliais, associadas a fenômenos inflamatórios, que geram progressivas lesões teciduais, culminando com fibrose. Sua etiologia é ainda desconhecida, porém deriva da interação entre predisposição genética, desregulação imunológica e estímulos ambientais. A resposta à imunossupressão convencional costuma ser pobre e os pacientes com acometimento cutâneo difuso e/ou envolvimento vis-

${ }^{l}$ Médica(o). Unidade de Transplante de Medula Óssea e Divisão de Doenças Neuromusculares, Hospital das Clínicas da FMRP-USP Ribeirão Preto-SP

${ }^{2}$ Médica(o). Serviço de Hematologia e Transplante de Medula Óssea, Hospital Israelita Albert Einstein, São Paulo- SP

Correspondência: Júlio C. Voltarelli

Centro Regional de Hemoterapia do Hospital das Clínicas

Faculdade de Medicina de Ribeirão Preto

Rua Tenente Catão Roxo, 2501

Tel./Fax.: (55 16) 2101-9369; Fax (55 16) 2101-9309

14051-140 - Ribeirão Preto-SP - Brasil

E-mail: jcvoltar@fmrp.usp.br

Doi: 10.1590/S1516-84842010005000023 
ceral, especialmente pulmonar, apresentam mortalidade que varia de $30 \%$ a $50 \%$ em cinco anos. ${ }^{3}$

O transplante de células-tronco hematopoéticas (TCTH) tem sido empregado com frequência crescente nos últimos 13 anos, embora de modo ainda experimental e voltado para os casos mais graves da doença. Dados provenientes dos registros internacionais de transplantes somam 175 pacientes transplantados no registro europe $u^{4}$ e 87 no registro americano (M. Pasquini, comunicação pessoal) até o ano de 2009. O procedimento tem sido aperfeiçoado e a incidência de mortalidade e de complicações graves diminuiu ao longo do tempo. Restam ainda muitos pontos de discussão, alguns dos quais deverão ser esclarecidos com os protocolos randomizados em andamento.

O presente consenso visa reunir os dados disponíveis na literatura internacional, confrontá-los com a experiência brasileira e estabelecer recomendações baseadas em evidências para a aplicação do TCTH para esclerose sistêmica no Brasil.

\section{Tratamentos convencionais}

A esclerose sistêmica representa um desafio às terapias convencionais disponíveis. Corticosteroides e imunossupressores, geralmente usados no tratamento de outras doenças reumáticas, com maior sucesso, pouco influenciam no curso desta doença. Mais recentemente, drogas antifibróticas, como a D-penicilamina, e antagonistas de receptores da endotelina, como a bosentana, também têm sido aplicadas na doença, porém com respostas limitadas. Outras drogas, como o micofenolato de mofetil e mesmo o imatinib e o dasatinib também vêm sendo usadas, ainda sem estudos conclusivos. ${ }^{5}$

Estudos randomizados, realizados nos últimos anos, demonstraram resultados conflitantes. Em 1996, van der Hoogen e colaboradores publicaram um estudo comparando metotrexato com placebo, no tratamento da esclerose sistêmica inicial. Houve melhora cutânea significativa no grupo tratado com metotrexato em baixas doses ( $15 \mathrm{mg} / \mathrm{semana})$, porém o tempo de seguimento foi curto, entre 24 e 48 semanas e somente $11 / 29(38 \%)$ dos pacientes apresentavam forma difusa da doença, havendo mais pacientes portadores da forma limitada no grupo placebo do que no grupo tratado com o metotrexato, respectivamente doze pacientes contra seis. ${ }^{6}$ Um estudo posterior, também randomizado e duplocego, avaliou 71 pacientes com forma inicial de esclerose sistêmica, com tempo de seguimento de um ano. Neste estudo, mais homogêneo, não houve diferenças entre os grupos, ao contrário do estudo anterior, sugerindo falta de eficácia do metotrexato para tratar a doença. ${ }^{3}$

Em 2002, um estudo inglês avaliou 14 pacientes com acometimento pulmonar intersticial pela esclerose sistêmica submetidos a pulsos mensais de ciclofosfamida e metilprednisolona. Tomografias computadorizadas de alta resolução e avaliações de difusão de $\mathrm{CO}$ evidenciaram melhora do quadro pulmonar após seis meses de pulsos, resposta que se manteve após mais seis meses de observação, suspensos os pulsos. Entretanto, em tempo médio de 26 meses, $67 \%$ dos pacientes tornaram a apresentar progressão do acometimento pulmonar. ${ }^{7}$ Em 2006, um estudo também realizado na Inglaterra, multicêntrico, prospectivo e duplo-cego, avaliou 45 pacientes com envolvimento pulmonar pela doença, divididos em dois grupos. O primeiro recebeu baixas doses de corticosteroides, mais seis pulsos mensais de ciclofosfamida, seguidos por azatioprina oral como manutenção, e o segundo recebeu placebo. Os pacientes foram acompanhados durante aproximadamente 12 meses, porém não houve diferenças significativas entre os grupos. ${ }^{8}$ Ainda no mesmo ano de 2006, um grupo norte-americano avaliou 145 pacientes com acometimento pulmonar, em estudo também randomizado, duplo-cego e prospectivo. Houve melhora estatisticamente significativa, mas discreta, da função pulmonar, do espessamento cutâneo e da qualidade de vida dos pacientes tratados com ciclofosfamida oral durante um ano., ${ }^{9,10}$

Devido à pobre resposta da doença à terapia convencional, que não é capaz de reverter a fibrose responsável pelas alterações funcionais, a mortalidade global da doença em cinco anos é de cerca de 30\%, subindo para $40 \%-50 \%$ em afetados por formas progressivas da doença. ${ }^{11}$

\section{Experiência internacional com transplante de células-tronco hematopoéticas para esclerose sistêmica}

Grande parte da informação sobre o transplante de células-tronco hematopoéticas (TCTH) para esclerose sistêmica provém dos estudos multicêntricos norte-americanos e europeus, não randomizados, que vêm sendo publicados desde 2001. O registro europeu de transplantes relatou, em 2004, sua experiência com 57 pacientes transplantados. ${ }^{12}$ Desses, 50 apresentavam forma cutânea difusa da doença e 40 algum tipo de acometimento pulmonar, na forma de pneumopatia intersticial ou hipertensão pulmonar. Apesar da grande variedade de regimes usados, predominaram os condicionamentos com ciclofosfamida em altas doses, associada ou não à globulina antilinfocitária (ATG). Aproximadamente $60 \%$ a $70 \%$ dos pacientes evoluíram com melhora cutânea significativa e duradoura e houve estabilização do envolvimento pulmonar. Cinco $(8,6 \%)$ pacientes morreram por causas associadas ao transplante e outros oito (14\%) por progressão da doença, em seguimento médio de 22 meses. Aproximadamente $35 \%$ dos pacientes apresentaram progressão da doença em média 10 meses pós-transplante. Quando os dados foram comparados com aqueles de uma primeira publicação do mesmo grupo, datada de 2001, observou-se melhora da sobrevida e diminuição da mortalidade relacionada ao transplante (MRT) ${ }^{12,13}$

O grupo multicêntrico norte-americano, liderado pelo Dr. R. Nash, do FHCRC/Seattle, publicou, em 2007, um 
conjunto de 34 pacientes com envolvimento cutâneo difuso, submetidos a condicionamento com irradiação corporal total (TBI), ciclofosfamida e ATG. ${ }^{14}$ Esse regime, mieloablativo, gerou algumas críticas a partir de outros grupos de transplante, que defendem esquemas menos tóxicos. ${ }^{15}$ Observouse melhora cutânea significativa e estabilização das funções pulmonar, renal e cardíaca. Neste estudo, de forma inédita, a melhora do acometimento cutâneo foi comprovada por biópsias comparativas de pele realizadas antes e seis meses após o transplante. Houve 12 óbitos durante o estudo, sendo oito relacionados ao transplante e quatro por progressão da doença. Estimaram-se a sobrevida livre de progressão e a sobrevida global em $64 \%$.

O centro de transplante da Northwestern University, em Chicago-IL, apresentou pequena casuística em publicação de 2007. ${ }^{16}$ Seus resultados, entretanto, são de grande relevância devido à grande experiência do grupo com TCTH para doenças autoimunes, especialmente por sua baixa taxa de óbitos relacionados ao procedimento. Foram relatados dez pacientes com acometimento difuso pela doença, associada ao comprometimento de pelo menos uma víscera, dentre pulmões, coração e trato gastrointestinal. Os pacientes foram submetidos a condicionamento não mieloablativo, com ciclofosfamida $120 \mathrm{mg} / \mathrm{kg}$ e ATG de coelho $7,5 \mathrm{mg} / \mathrm{kg}$, seguido por infusão de células autólogas não selecionadas in vitro. Todos os pacientes apresentavam envolvimento gastrointestinal e pneumopatia intersticial, mas nenhum apresentava hipertensão pulmonar ou alterações renais significativas. Todos apresentaram melhora cutânea inicial, mas dois evoluíram com progressão do acometimento após o transplante. Todos os pacientes estabilizaram suas funções pulmonares, cardíacas e renais. Houve um único óbito, não relacionado ao transplante. A sobrevida global foi de $90 \%$ e a livre de progressão foi de $70 \%$.

O maior tempo de seguimento foi relatado, em 2008, pela associação de um centro francês e dois centros holandeses. ${ }^{17}$ Nesse estudo, 26 pacientes foram submetidos a transplante autólogo, todos condicionados com $200 \mathrm{mg} / \mathrm{kg}$ de ciclofosfamida e resgatados com células previamente selecionadas, para enriquecimento de células CD34+. Doze pacientes apresentavam acometimento cutâneo difuso, e 14, envolvimento visceral, principalmente pulmonar. Em um seguimento médio de 5,3 anos, $81 \%$ dos pacientes apresentaram melhora clínica com o transplante. Houve também melhora significativa do acometimento cutâneo em 94\% dos pacientes e estabilização das funções pulmonar, renal e cardíaca. A sobrevida global estimada por curva de Kaplan-Meier foi de 96,2\% em cinco anos e $86,8 \%$ em sete anos. Seis (28\%) dos pacientes apresentaram reativação da doença após o transplante, necessitando de tratamento imunossupressor adicional. Desses, somente um evoluiu com progressão da doença.

Atualmente, dois grandes estudos randomizados encontram-se em andamento, visando avaliar comparativamente os efeitos do transplante autólogo para esclerose sistêmica em relação ao tratamento convencional. O estudo ASTIS, europeu, vigente desde 2002, compara pulsos de ciclofosfamida com o transplante autólogo realizado com drogas linfoablativas: $200 \mathrm{mg} / \mathrm{kg}$ de ciclofosfamida e ATG de coelho. Até publicação de $2008,{ }^{18} 122$ pacientes haviam sido incluídos, sendo 44 no braço do transplante e outros 48 no braço controle, e o transplante vinha se mostrando seguro, com somente um óbito relacionado ao procedimento. Resultados de eficácia ainda não foram divulgados. O estudo SCOT, multicêntrico, norte-americano, é mais recente e, até 2007 , havia incluído 26 pacientes. Compara pulsos de ciclofosfamida com transplante autólogo mieloablativo, que inclui 800 cGy de TBI acrescida de $120 \mathrm{mg} / \mathrm{kg}$ de ciclofosfamida e ATG de cavalo (hATG). Futuramente, esses estudos trarão respostas quanto à real eficácia do transplante em relação ao tratamento convencional. ${ }^{19}$

Enquanto os resultados desses estudos prospectivos randomizados não são disponíveis, os resultados dos estudos de fase II do TCTH têm que ser comparados com os de ensaios clínicos usando outras formas de imunossupressão, como ciclofosfamida oral ou em pulsos endovenosos e metotrexato (revisados na ref 19). Esses estudos, em geral realizados com grupos pequenos de pacientes, mostraram resultados positivos na estabilização das manifestações pulmonares e cutâneas de pacientes com formas progressivas da doença.

\section{Pontos de discussão}

A experiência adquirida com os TCTH para esclerose sistêmica desde o início de sua aplicação, em 1996, tem evidenciado algumas dificuldades no manejo da doença durante o procedimento. Entre as doenças autoimunes, a esclerose sistêmica apresenta uma das maiores taxas de mortalidade relacionadas ao transplante, muito provavelmente devido às más condições clínicas dos pacientes, em especial cardiopulmonares. Atualmente, existe uma grande preocupação em selecionar adequadamente o paciente a ser transplantado, bem como detectar disfunções cardíacas subclínicas. Além disso, alguns pontos de divergência em relação à metodologia do transplante têm surgido entre os principais pesquisadores, exigindo uma cuidadosa avaliação da literatura disponível.

As principais pendências em relação ao TCTH para esclerose sistêmica estão listadas a seguir, cada qual acompanhada dos dados de literatura disponíveis.

\section{Condicionamento mieloablativo ou não mielo-} ablativo?

Na publicação do grupo americano multicêntrico, todos os pacientes receberam condicionamento mieloablativo, com irradiação corporal total (TBI) e globulina antilinfocitária de cavalo. ${ }^{14}$ Tal regime foi criticado pelo grupo de Chicago, que, em sua experiência, considerou o uso de um esquema não 
mieloablativo igualmente eficaz e menos tóxico, considerando a labilidade cardiorrespiratória desse grupo de pacientes. ${ }^{15}$ No grupo multicêntrico europeu, houve maior variação entre os regimes de condicionamento, porém, dentre os 57 pacientes incluídos no estudo, pelo menos 47 foram condicionados com ciclofosfamida em altas doses (não mieloablativo), com ou sem ATG. ${ }^{12,13}$ Os três estudos apresentaram resultados semelhantes quanto ao controle da doença, porém, o estudo mieloablativo apresentou mortalidade relacionada ao transplante (TRM) de $23,4 \%$, enquanto nos não mieloablativos a TRM foi de 8,6\% no grupo multicêntrico europeu e zero no grupo de Chicago. Regimes menos mieloablativos, portanto, poderiam favorecer o paciente, diminuindo a toxicidade do TCTH sem comprometer sua eficácia, embora maior número de casos e maior tempo de seguimento sejam necessários.

Qual regime de condicionamento não mieloablativo? Considerando os regimes não mieloablativos, os TCTH para doenças autoimunes empregam, em sua grande maioria, ciclofosfamida em altas doses. A vasta experiência com a droga tem superado a curva de aprendizado, o que contribui para a redução das complicações relacionadas ao transplante. A ciclofosfamida, no entanto, é conhecidamente cardiotóxica, e pode gerar um quadro dose-dependente de edema e hemorragia miocárdica, que se manifesta por insuficiência cardíaca grave, aguda e potencialmente fatal. ${ }^{20}$ Os pacientes com comprometimento cardíaco deverão, portanto, ser avaliados individualmente quanto à possibilidade de substituição da droga ou mesmo suspensão do transplante. Esquemas contendo fludarabina e melfalan também podem se associar a toxicidade cardíaca, embora não existam descrições de eventos tóxicos em transplantes para doenças autoimunes. ${ }^{21}$ Komatsuda e colaboradores descreveram o TCTH aplicado a um paciente com comprometimento cardíaco, usando thiotepa e ciclofosfamida em doses reduzidas $(100 \mathrm{mg} / \mathrm{kg})$ como regime de condicionamento. Não houve toxicidade durante o transplante e o paciente obteve remissão da doença. ${ }^{22}$

Como avaliar a função cardíaca do paciente com esclerose sistêmica?

$\mathrm{Na}$ esclerose sistêmica pode haver comprometimento do sistema de condução cardíaca ou da contratilidade cardíaca, além de hipertensão pulmonar e quadros, menos comuns, de coronariopatias. O comprometimento, principalmente a disfunção contrátil, pode ser subclínica e não ser detectada através dos exames convencionais de avaliação pré-transplante, como eletrocardiograma ou ecocardiograma. Recentemente, algumas publicações têm abordado o tema, propondo algoritmos de avaliação pré-transplante, visando detectar os pacientes de alto risco. ${ }^{20,23}$ Miniati e cols propuseram, em 2007, uma sequência de passos a serem seguidos na investigação de disfunções cardíacas em portadores de esclerose sistêmica. ${ }^{20} \mathrm{O}$ primeiro passo seria uma cuidadosa avaliação clínica cardíaca, acompanhada por ECG e radiografia de tórax. A seguir, ecocardiograma, dosagem de peptídeo atrial natriurético e curva de troponina, para avaliar acometimento de miocárdio, além de Holter para avaliar distúrbios de condução. Em caso de alterações nas etapas anteriores, seguir-se-iam ventriculografia de esforço e mesmo cateterismo cardíaco, este último caso áreas isquêmicas fossem detectadas.

\section{Transplante autólogo ou alogênico?}

O transplante autólogo tem sido preferido em relação ao alogênico devido à sua maior segurança; entretanto, a porcentagem de reativações da doença é fator preocupante, já que, aproximadamente, $30 \%$ dos pacientes têm progressão da doença após o transplante. Pesquisadores estudam maneiras de melhorar o controle da doença, sem, no entanto, aumentar o risco do procedimento. No ano de 2008, um grupo japonês relatou sua experiência com transplante de células-tronco alogênicas para uma paciente portadora de esclerose sistêmica. ${ }^{24}$ Houve melhora cutânea e estabilização da função pulmonar, embora a paciente tenha desenvolvido doença do enxerto-versus-hospedeiro renal, na forma de glomerulopatia membranosa, que foi responsiva ao tratamento com corticosteroides. Em 2007, de modo semelhante, Loh e colaboradores publicaram os resultados de um transplante alogênico não mieloablativo para uma paciente portadora de acometimento cutâneo difuso e hipertensão pulmonar. ${ }^{25}$ Após o transplante, que transcorreu sem intercorrências e sem desenvolvimento de GVHD, houve melhora cutânea e diminuição dos níveis de pressão de artéria pulmonar.

Alguns argumentos favorecem o transplante alogênico em comparação com o autólogo, como o efeito GVA (graftversus-autoimmunity), em que linfócitos do doador manteriam uma vigilância imunológica, destruindo possíveis linfócitos autorreativos do receptor. Outro argumento seria o de que as novas células imunológicas, após o transplante, seriam originadas a partir de células-tronco não suscetíveis geneticamente à doença, reduzindo o risco de reativação da esclerose sistêmica. Embora os casos descritos tenham mostrado resultados animadores para os transplantes alogênicos, ainda são insuficientes para comprovar segurança e superioridade aos transplantes autólogos. Um novo estudo randomizado (ALLSTAR) foi planejado nos Estados Unidos, patrocinado pelo NIH, visando avaliar a eficácia do transplante alogênico para esclerose sistêmica em comparação com o melhor tratamento convencional disponível, excluindo o transplante autólogo. ${ }^{19}$ Entretanto, esse estudo foi suspenso pelo NIH antes de ter se iniciado.

Realizar depleção in vitro de células T?

Muitos centros selecionam as células infundidas no paciente, eliminando células T e concentrando células CD34+. 
Nem todos os pesquisadores, entretanto, concordam com o procedimento. ${ }^{26}$ Além de encarecer o transplante, a seleção reduz o número de células-tronco disponíveis para infusão e predispõe o material a possíveis contaminações, pela manipulação à qual é submetido. ${ }^{27}$ Seus benefícios, além disso, são questionáveis. Snowden e cols observaram, em um estudo multicêntrico europeu, que pacientes portadores de artrite reumatoide, transplantados com ou sem depleção de células $\mathrm{T}$, apresentavam a mesma taxa de remissão, ${ }^{28} \mathrm{e}$, em um estudo australiano, a seleção de células CD34+ foi associada a menor taxa de resposta ao TCTH para AR.${ }^{29}$ Vários centros de transplante, como o de Chicago, Estados Unidos, estão dispensando a depleção de células $\mathrm{T}$ de seus protocolos mais recentes.

Pacientes com acometimento cutâneo exclusivo devem ser transplantados?

Os TCTH para esclerose sistêmica têm demonstrado grande impacto sobre a pele dos pacientes, com importante redução da fibrose cutânea, o que se associa com recuperação da amplitude de movimentos articulares, diminuição da incidência de úlceras de extremidades e melhora da qualidade de vida. ${ }^{12-19}$ Adicionalmente, pacientes com acometimento difuso da pele, mesmo que isolado, sem envolvimento visceral, apresentam baixa sobrevida de longo prazo, justificando os riscos do transplante. ${ }^{30}$

O TCTH reverte alterações isquêmicas da microcirculação?

Deficiências de perfusão de extremidades e úlceras digitais são manifestações frequentes e debilitantes nos pacientes com esclerose sistêmica. Miniati e cols. descrevem reversão do comprometimento capilar de leito ungueal, mostrando angiogênese e revascularização de áreas antes isquêmicas em seis pacientes transplantados. ${ }^{31} \mathrm{O}$ efeito angiogênico das células-tronco foi também demonstrado por Nevskaya e cols, em 2009, em estudo de injeções locais de células provenientes de medula óssea ou sangue periférico em palmas das mãos e panturrilhas de pacientes com grave comprometimento da circulação e úlceras de extremidades. ${ }^{32}$ Os pacientes evoluíram com importante melhora da irrigação dos membros tratados e fechamento de grande parte das úlceras isquêmicas.

Como tratar as progressões pós-transplante?

De acordo com a literatura, aproximadamente $30 \%$ dos pacientes transplantados apresentam reativação da doença pós-transplante. Vonk e cols sugerem imunossupressão com corticosteroides, micofenolato de mofetil e/ou ciclosporina como possíveis tratamentos, porém seus resultados não foram divulgados. ${ }^{17}$ Submeter o paciente a um segundo transplante também é uma opção a ser considerada, porém, ao contrário de outras doenças autoimunes, não há descrições em literatura.
O TCTH é superior ao tratamento convencional com pulsos mensais de ciclofosfamida?

Em 2005, um grupo francês descreveu uma comparação retrospectiva entre pacientes com envolvimento pulmonar pela esclerose sistêmica, divididos em grupos tratados com pulsos mensais de ciclofosfamida ou com TCTH autólogo, usando ciclofosfamida em altas doses. ${ }^{33}$ Concluíram que a toxicidade entre os esquemas era semelhante, porém a eficácia dos dois regimes deverá ser mais bem avaliada pelos estudos randomizados, em andamento. Antes da conclusão desses estudos, entretanto, existem pacientes com marcadores de muito mau prognóstico da doença e aqueles que não responderam aos pulsos mensais com ciclofosfamida e que, portanto, têm indicação de TCTH, baseando-se na experiência acumulada com esse procedimento.

\section{Experiência nacional}

Casos esporádicos de transplante para esclerose sistêmica foram realizados desde 1999, porém somente a partir de 2005 o procedimento tem sido aplicado com maior frequência e de modo mais padronizado. No Hospital das Clínicas de Ribeirão Preto (HCRP-USP), foram transplantados, até o início de 2009, 12 pacientes com acometimento cutâneo difuso e progressivo. Desses, sete apresentavam envolvimento pulmonar, e seis, acometimento significativo de trato gastrointestinal, com algum grau de desnutrição proteico calórica. Seis, dos doze pacientes, haviam sido tratados previamente com pulsos de ciclofosfamida, sendo quatro portadores de envolvimento pulmonar. Uma paciente desistiu do transplante devido a melhora cutânea e pulmonar logo após a mobilização com ciclofosfamida, e uma paciente evoluiu para óbito, ainda na fase de mobilização, por pneumonia aspirativa. Uma paciente apresentou insuficiência cardíaca congestiva durante o transplante, e as avaliações laboratoriais e clínicas constataram disfunção diastólica grave, também relacionada à esclerose sistêmica. Oito pacientes apresentaram neutropenia febril e dois desenvolveram pneumonia bacteriana, resolvida com antibióticos. Em seguimento médio de aproximadamente 18 meses (2-30), dois pacientes apresentaram progressão da doença e os demais apresentaram melhora cutânea. A sobrevida livre de progressão, no período, foi de $66 \%$, e a sobrevida global foi de $91,6 \% .{ }^{34}$ Outros casos, em menor número, também foram transplantados em outros centros do Brasil, com resultados semelhantes. ${ }^{35}$

\section{Conclusões}

A sequência de publicações internacionais revela que o transplante autólogo vem se consolidando como alternativa terapêutica para a esclerose sistêmica. Seus resultados, principalmente em relação ao controle do acometimento pulmonar, são animadores, pois atinge respostas até agora não obtidas com outras formas de tratamento. Adicio- 
nalmente, observa-se que a mortalidade e a frequência de eventos adversos relacionados ao procedimento vêm diminuindo conforme cresce a experiência e o número de pacientes transplantados. Acredita-se que este resultado se deva à seleção mais criteriosa dos pacientes, excluindo aqueles que apresentam comorbidades e disfunções de órgãos secundárias à doença. No Brasil, os resultados, embora mais recentes, assemelham-se aos da literatura internacional, assim como são semelhantes as dúvidas e divergências em relação ao procedimento.

Com base nas evidências bibliográficas disponíveis e na experiência nacional, que consistem em estudos clínicos não randomizados, concluímos que, para o tratamento da esclerose sistêmica, o transplante autólogo de TCTH seja classificado como nível de recomendação A-II e que ele seja indicado, no presente estágio do conhecimento, para formas progressivas da doença com acometimento cutâneo difuso ou visceral grave. Esta indicação se fundamenta na alta mortalidade e baixa qualidade de vida dos pacientes com ES acometidos por essas formas da doença e na resposta insatisfatória aos tratamentos medicamentosos disponíveis. Os estudos randomizados ASTIS e SCOT, em andamento, terão fundamental importância para fornecer dados mais consistentes, eventualmente elevando os graus de evidência e recomendação do transplante autólogo para esclerose múltipla no futuro.

\section{Indicações de transplante de células-tronco hematopoéticas para esclerose múltipla}

A esclerose múltipla (EM) é uma doença inflamatória crônica e desmielinizante do sistema nervoso central, que afeta principalmente adultos jovens. Estima-se que, atualmente, cerca de 300 mil norte-americanos sejam portadores da doença. A mortalidade em pacientes com EM não difere muito da encontrada na população normal, porém a progressão dos déficits neurológicos ocorre em todos os portadores da doença. Avalia-se que, 15 anos após o início da doença, cerca de $50 \%$ dos pacientes necessitam de auxílio para deambular e, após 25 anos, a maioria está incapacitada para andar. Essas incapacidades parecem estar relacionadas com o número e gravidade dos surtos nos primeiros anos da doença, daí a importância de um diagnóstico e tratamento precoces.

O quadro histopatológico da EM caracteriza-se pela presença de placas de desminielização, localizadas preferencialmente em torno dos ventrículos, nos nervos ópticos e no cérebro. No surtos, observa-se nas placas infiltrado perivascular de linfócitos.

A maioria dos pacientes apresenta um quadro clínico com surtos que se alteram com remissões. Os sintomas da doença são inespecíficos: parestesias, paresias, ataxia, tremor, neurite óptica, fadiga e alterações esfincterianas. O exame neurológico revela alterações objetivas compatíveis com a disseminação da doença por comprometimento da substância branca, no tempo e no espaço. Considera-se surto a ocorrência de sintomas de disfunção neurológica, incluindo dados de anamnese, com mais de 24 horas de duração, na ausência de febre ou infecção e com comprometimento objetivo de pelo menos dois sistemas de fibras longas (substância branca). No exame neurológico, durante o surto, ocorre piora neurológica, isto é, aumento do escore da escala de incapacidade funcional. Durante a remissão, o paciente permanece estável dentro da sua escala de incapacidade. Inúmeras escalas foram descritas, sendo a mais usada a Escala Expandida de Incapacidade Funcional (EDSS), descrita por Kurtzke.

Vários são os critérios diagnósticos existentes, porém os critérios de Poser e de McDonald são os mais utilizados nos ensaios terapêuticos. Os critérios propostos por Poser incluem, além dos dados clínicos, exames complementares, tais como ressonância nuclear magnética e exame do líquido cefalorraquidiano (LCR).

A esclerose múltipla pode ser classificada em três formas clínicas principais: a) forma remitente-recorrente, que se caracteriza por surtos episódicos seguidos de recuperação parcial ou total da disfunção, intercalados por períodos de remissão de, no mínimo, trinta dias. Após um tempo médio de dez anos, os surtos tornam-se menos frequentes, seguidos de recuperação menos evidente, com acúmulo de sequelas e piora gradativa do quadro neurológico, caracterizando a forma secundariamente progressiva; $b$ ) Alguns pacientes iniciam o quadro com sintomas e sinais progressivos desde o início da doença, caracterizando a forma primariamente progressiva; c) Também tem sido reconhecida uma "forma maligna" de esclerose múltipla, termo este aplicado a casos severos de EM, com um curso clínico rapidamente progressivo para uma forma severa de incapacidade neurológica ou, até mesmo, morte em um curto período de tempo.

Embora sua etiologia não esteja totalmente definida, várias evidências, como a presença de bandas oligoclonais no LCR, sugerem que um mecanismo de autoimunidade esteja envolvido na patogênese da EM.

\section{Experiência no uso de transplante de células- tronco hematopoéticas na esclerose múltipla}

Um dos primeiros trabalhos publicados de utilização de TCTH em EM foi o de Fassas e cols, em 1997, ${ }^{36}$ relatando o resultado desta modalidade terapêutica em 15 pacientes. Os pacientes receberam quimioterapia em altas doses, globulina antilinfocitária de coelho na dose de $5 \mathrm{mg} / \mathrm{kg} / \mathrm{dia}$, após infusão das CTH e não foi realizada nenhuma manipulação do material coletado para depleção da população linfoide. Com um tempo médio de seguimento de apenas seis meses, 12 pacientes apresentaram melhora do quadro clínico. Kosak e cols também publicaram sua experiência com oito pacientes com EM, que receberam quimioterapia em altas doses. Em sete pacientes, o material coletado foi submetido a depleção ex vivo de células $\mathrm{T}$, e apenas um deles recebeu 
globulina antilinfocitária devido ao número insuficiente de células CD34+ coletado. Apenas um paciente não apresentou resposta ao TCTH, com estabilização do seu quadro neurológico. Um paciente melhorou significativamente, cerca de 1,5 ponto na escala EDSS, e dois pacientes apresentaram melhora discreta: 0,5 ponto.

O relato do Grupo Europeu de Transplante de Medula Óssea, em 1999, descreveu os resultados de 74 pacientes submetidos a transplante autólogo para doenças autoimunes, sendo 35 doenças reumáticas, 31 neurológicas, 5 hematológicas e 3 vasculites. Em todos os pacientes houve enxertia, 5 morreram e, dos 60 avaliáveis, 40 obtiveram algum tipo de melhora. Em 2002, a atualização deste relatório mostrou que o número de pacientes submetidos a transplante autólogo atingiu o total de 251, sendo que, destes, 149 tinham o diagnóstico de doença reumatológica, 91 com doenças neurológicas, $15 \mathrm{com}$ doenças hematológicas. Deste total, 220 receberam transplantes de células obtidas de sangue periférico, 40 de medula óssea e, em um paciente, foram coletados e infundidos ambos. Em 66 pacientes não foi realizada nenhuma manipulação para depleção celular no material colhido para resgate hematopoético. Dos 89 pacientes submetidos a transplante autólogo para EM, descritos neste relatório, $67 \%$ apresentaram estabilização ou melhora do quadro num tempo de seguimento médio de 13 meses. A mortalidade relacionada ao tratamento foi de 5,6\%. Em 2006, o número de pacientes com EM transplantados pelo grupo europeu aumentou para 178 , sendo que, em $63 \%$ dos casos, mostraram-se estáveis ou com melhora, e a mortalidade relacionada à terapia foi de 5,3\%. Em 2009, no congresso europeu de transplante, Saccardi, em apresentação oral, mostrou nova atualização da experiência do EBMT em doenças autoimunes. Foram realizados 1.078 transplantes em doenças autoimunes; desses, $386 \mathrm{em}$ EM. A sobrevida global de todos os pacientes em cinco anos foi de $85+/-1 \%$. Na EM, em três anos de seguimento, a sobrevida global foi de $93+/-2 \%$. A sobrevida livre de progressão geral ao cinco anos foi de $43+/-4 \%$, e para EM, em três anos, foi de $55+/-3 \%$.

Após os estudos pioneiros iniciados em 1995, mais de 400 casos de EM haviam sido tratados no mundo. A Tabela 1 resume o resultado da experiência em TCTH em EM dos maiores centros no mundo.

Esses estudos foram analisados recentemente em uma revisão de Fassas e Mancardi, ${ }^{54}$ que concluíram que "esses resultados (de TCTH) parecem superiores aos produzidos pela terapia convencional, mas esta superioridade precisa ser demonstrada em estudos comparativos de fase III, como o ASTIMS lançado pelo EBMT". Entretanto, este estudo, devido ao baixo recrutamento de pacientes, foi convertido em um estudo de fase II e não dará a resposta pretendida. Continuando "O TCTH não é um tratamento para a população geral de pacientes com EM, mas para um grupo selecionado de pacientes que não respondem à terapia convencional e progridem rapidamente. TCTH deve ser usado precocemente, durante a fase inflamatória da doença, usando regimes de condicionamento de intensidade intermediária. Os resultados do TCTH são dramáticos e salvam vidas nos casos de EM maligna".

Recentemente, o grupo brasileiro de transplante em doença autoimune fez a análise de três anos da experiência com o uso de TCTH em esclerose múltipla onde comparou dois tipos de condicionamento, BEAM/ATG e ciclofosfamida/ ATG. ${ }^{55}$ Foram realizados, neste protocolo, 41 transplantes autólogos. Os resultados mostraram uma sobrevida livre de eventos global de $58,54 \%$ e, quando analisados separadamente, foi de $47 \%$ no grupo BEAM/ATG e $70 \%$ no grupo ciclofosfamida/ATG sem diferença estatística $(p=0.288)$. Houve três óbitos no grupo BEAM e nenhum no grupo CI. Após o encerramento desse estudo, outros pacientes foram submetidos a TCTH usando CI/ATG de coelho no condicionamento, totalizando 46 pacientes no HCRP e com resultados semelhantes na evolução e nenhuma mortalidade. O seguimento neurológico, com tempo médio de 26 meses ( 3 a 52), em 38 pacientes com seguimento maior que seis meses, mostrou melhora em $30 \%$ dos pacientes, estabilização em $47 \%$ e piora em $23 \%$. Esses dados, apesar do pouco tempo de avaliação, mostram a possibilidade de uso de regimes de condicionamento de baixa intensidade no condicionamento.

\section{Indicação de transplante de células-tronco hematopoéticas autólogo em esclerose múltipla}

A introdução de imunomoduladores na terapêutica da EM, a partir de 1993, modificou o cenário da EM. Atualmente o tratamento da EM inclui primariamente imunomoduladores (beta-interferon, copolímero, imunoglobulinas), e terapia com corticosteroides para a fase aguda de recidiva (surtos) e, após isso, imunossuppressores (ciclosporina, ciclofosfamida, azatioprina, mitoxantrone, etc). Estudos recentes de um antagonista seletivo da alfa-4 integrina, natalizumab, mostraram resultados promissores na redução do risco de progressão e recidiva clínica em pacientes com EM recidivada quando comparada com placebo, como primeira linha de tratamento e também como segunda linha na forma remitenterecorrente da EM. O uso do rituximab e do alentuzumab também apresenta redução nas lesões cerebrais inflamatórias e recidiva clínica na forma remitente-recorrente da EM. Atencão deve ser dada ao aumento da incidência de infecções com o uso destas drogas imunussupressoras e o surgimento de leucoencefalopatia posterior reversível com o uso de natalizumab.

A doença geralmente se inicia com surtos seguidos de remissão (relapsing-remitting - RR), que é seguida, 5-15 anos depois, por uma fase secundária progressiva, com ou sem surtos e, após este período, aproximadamente $50 \%$ dos pacientes são incapazes de deambular sem ajuda. A sobrevida média de pacientes com EM é cerca de dez anos menor do que a população geral e o risco de mortalidade é aumentado 
Tabela 1. Revisão da literatura do tratamento da esclerose múltipla com transplante de células-tronco hematopoéticas

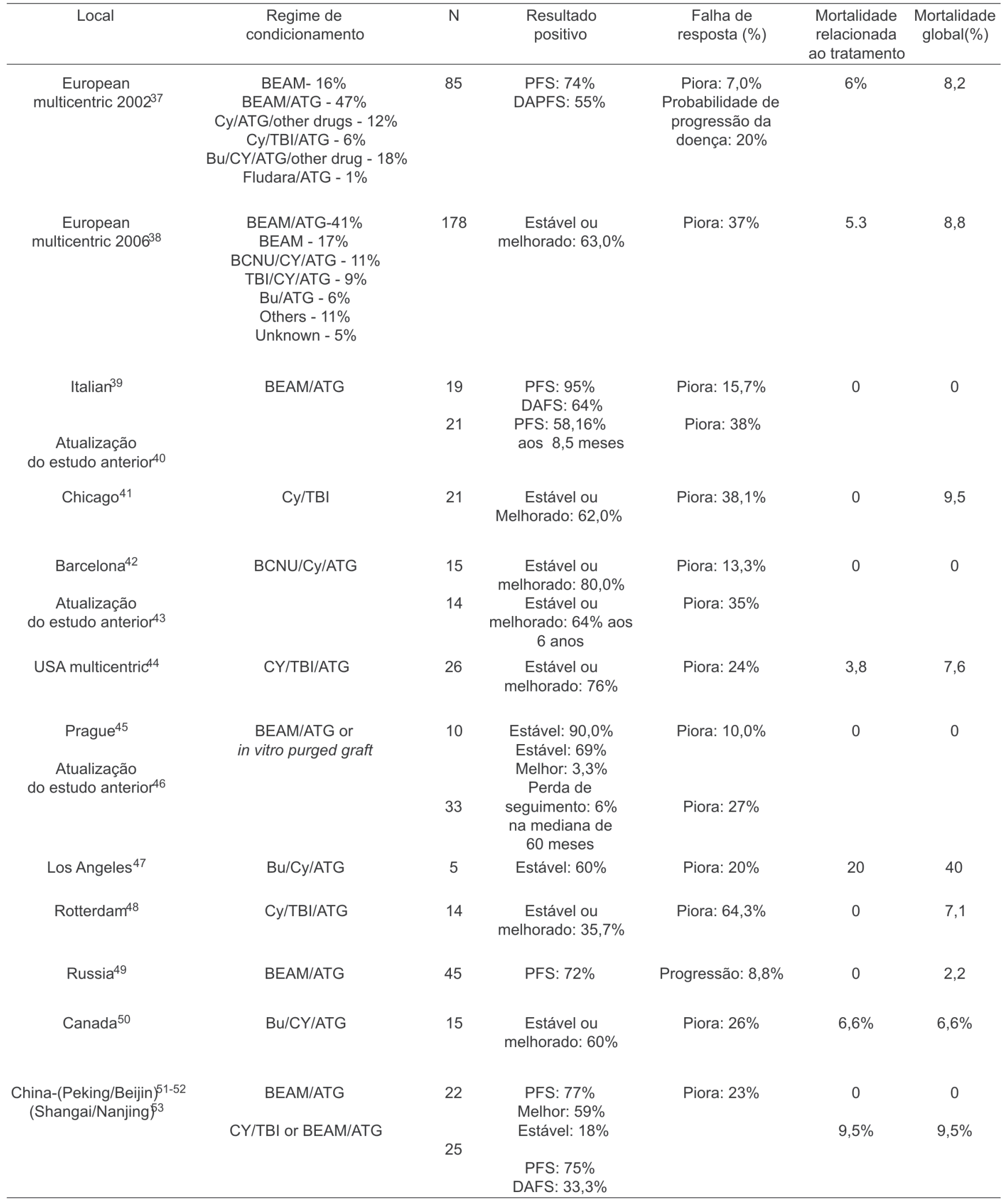

BEAM = BCNU, citocina, arabinosídeo, melfalan, etoposídeo; $A T G$ = globulina antilinfocitária; $C y=$ ciclofosfamida; TBI = irradiação corporal total; $\mathrm{Bu}$ = bussulfano; Fludara = fludarabina; PFS: sobrevida livre de progressão; DAPFS: sobrevida livre de progressão com doença ativa; DAFS: sobrevida livre de doença ativa. 
três vezes. Esses dados justificam a adoção de estratégias terapêuticas inovadoras para a doença.

Frente aos relatos recentes nesta área, o uso do TCTH pode ser uma opção promissora no tratamento da EM, já que tem a capacidade de suprimir completamente a inflamação e pode parar a progressão em aproximadamente $60 \%-70 \%$ dos pacientes com quadros severos de EM por um longo período de tempo. É também uma terapia poderosa para a "forma maligna" de EM e pode induzir uma profunda mudança no sistema imune por um período de ao menos dois anos. O limitante do TCTH é que não é uma terapia curativa, é um tratamento que carrega certa toxicidade com uma taxa de mortalidade geral, na análise dos estudos recentes, em torno de $1 \%-2 \%$.

De acordo com os estudos citados anteriormente, os pacientes que mais frequentemente se beneficiariam com TCTH são pacientes mais jovens ainda em estado ambulante (máximo de $\mathrm{EDSS}^{6,5}$ ), com a forma remitente-recorrente ou secundária progressiva que tenham falhado à terapia convencional aprovada com imunomoduladores (interferon-beta, acetato de glatiramer) e também falhado ou recidivado durante a terapia com imunossupressores (ciclofosfamida, mitoxantrone) e anticorpos monoclonais, quando acessíveis, e com presença de inflamação ao exame de ressonância magnética; também pacientes com a "forma maligna" de EM com rápida deterioração e aumento da incapacidade neurológica, que tenham falhado a todas as opções terapêuticas existentes (incluindo plasmaférese).

Pacientes muito comprometidos pela incapacidade neurológica (EDSS >6,5) com um curso estabelecido progressivo sem recidivas e sem atividade inflamatória na ressonância magnética não devem ser tratados com TCTH.

\section{Conclusão}

Os dados de literatura e da experiência brasileira apresentados respaldam a evidência científica do uso do transplante de células-tronco hematopoéticas em pacientes portadores de esclerose múltipla em progressão e sem resposta aos tratamentos imunomoduladores convencionais, como corticoesteroides, imunoglobulinas, interferon e co-polímeros. O papel da terapia biológica (Campath ${ }^{\circledR}$, natalizumab) na esclerose múltipla também mostra evidência de resposta, mas não há, ainda, comparação com o TCTH e esta terapia não é accessível à maioria da população .

Dessa forma, o consenso de TCTH em doenças autoimunes estabelece a indicação de transplante autólogo de células-tronco hematopoéticas em pacientes com esclerose múltipla progressiva não responsiva à terapêutica convencional para pacientes com EDSS entre 3.0 e 6.5. As formas da doença que podem se beneficiar do transplante são as de surto-remissão, primária ou secundária progressiva, desde que haja evidências inflamatórias no momento da indicação do transplante. O TCTH está indicado também na forma maligna da EM, que é rapidamente progressiva e sabidamente irresponsiva a qualquer forma de tratamento.

\begin{abstract}
In this paper, international literature and national experience on hematopoietic stem cell transplantation (HSCT) for autoimmune diseases were reviewed. Cumulative evidence indicates that autologous HSCT may benefit patients with inflammatory multiple esclerosis, refractory to available drug therapy, and progressive forms of diffuse cutaneous systemic sclerosis with or without systemic involvement. Initially, this treatment should be available in reference centers of the public health system, with experience in performing HSCT and in treating severe systemic autoimmune diseases. Rev. Bras. Hematol. Hemoter. 2010;32(Supl. 1):125-135.
\end{abstract}

Key word: Hematopoietic stem cell transplantation; autoimmune diseases; multiple sclerosis; systemic sclerosis.

\section{Referências Bibliográficas}

1.Voltarelli JC, Rodrigues MC, Moraes DM, Malmegrim KCR, Leal AM. Transplante de células-tronco hematopoéticas para doenças autoimunes e inflamatórias. Em "Transplante de células-tronco hematopoéticas", editado por JC Voltarelli, R Pasquini e Euza TT Ortega, Ed Atheneu, São Paulo, 2009, p. 456-498.

2. Couri CE, Oliveira MCB, Stracieri ABPL, Moraes DA, Pieroni F, Barros GMN, et al. C-peptide levels and insulin independence following autologous nonmyeloablative hematopoietic stem cell transplantation in newly diagnosed type 1 diabetes mellitus. JAMA 2009;301(15):1573-9.

3. Pope JE, Bellamy N, Seibold JR, Baron M, Ellman M, Carette S, et al. A randomized, controlled trial of methotrexate versus placebo in early diffuse scleroderma. Arthritis Rheum 2001; 44(6):1351-58.

4. Farge D, Labopin M, Tyndall A, Fassas A, Mancardi GL, van Laar J, et al. Autologous hematopoietic stem cell transplantation (HSCT) for autoimmune diseases: na observational study on 12 years of experience from the European Group for Blood and Marrow Transplantation (EBMT) Working Party on Autoimmmune Diseases. Haematologica, 2009, epub ahead of print.

5. Au K, Khanna D, Clements PJ, Furst DE, Tashkin DP. Current concepts in disease-modifying therapy for systemic sclerosisassociated interstitial lung disease: lessons from clinical trials. Curr Rheumatol Rep. 2009;11(2):111-9.

6. van der Hoogen FH, Boerbooms AM, Swaak AJ, Rasker JJ, van Lier HJ, van de Putte LB. Comparison of methotrexate with placebo in the treatment of systemic sclerosis: a 24 week randomized double-blind trial, followed by a 24 week observational trial. Br J Rheumatol. 1996;35(4):364-72.

7. Griffiths B. Miles S, Moss H, et al. Systemic sclerosis and intersticial lung disease: a pilot study using pulse intravenous methylprednisolone and cyclophosphamide to assess the effect on high resolution computed tomography scan and lung function. J Rheumatol. 2002; 29(11):2371-8.

8. Hoyles RK, Ellis RW, Wellsbury J, Lees B, Newlands P, Goh NS, et al. A multicenter, prospective, randomized, double-blind, placebo controlles trial of corticosteroids and intravenous cyclophosphamyde followed by oral azathioprine for the treatment of pulmonary fibrosis in scleroderma. Arthritis Rheum 2006;54(12):3962-70. 
9. Khanna D, Yan X, Tashkin D, Furst DE, Elashoff R, Roth MD, et al. Impact of oral cyclophosphamide on health-related quality of life in patients with active scleroderma lung disease: results from the scleroderma lung study. Arthritis Rheum. 2007; 56(5):1676-84

10. Tashkin D, Elashoff R, Clements PJ, Goldin J, Roth MD, Furst DE, et al. Cyclophosphamide versus placebo in scleroderma lung disease. N Engl J Med. 2006;354(25):2655-66.

11. Scussel-Lozentti L, Joyal F, Raynaud JP, Roussin A, Rich E, Goulet JR et al. Predicting mortality in systemic sclerosis: Analysis of a cohort of 309 French Canadian patients with emphasis on features at diagnosis as predictive factors for survival. Medicine (Baltimore). 2002;81(2):154-67.

12. Farge D, Passweg J, van Laar JM, Marjanovic Z, Besenthal C, Finke J, et al. Autologous stem cell transplantation in the treatment of systemic sclerosis: report from EBMT/EULAR Registry. Ann Rheum Dis. 2004;63(8):974-81.

13. Binks M, Passweg JR, Furst D, et al. Phase I/II trial of autologous stem cell transplantation in systemic sclerosis: procedure related mortality and impact on skin disease. Ann Rheum Dis 2001;60(6):577-84.

14. Nash RA, McSweeney PA, Crofford LJ, Sullivan KM, Furst DE, et al. High-dose immunosuppressive therapy and autologous hematopoietic cell transplantation for severe systemic sclerosis: long- term follow-up of the US multicenter pilot study. Blood. 2007;110(4):1388-96.

15. Burt RK, Kallunian K, Patel D, et al. The rationale behind autologous autoimmune hematopoietic stem cell transplant conditioning regimens: concerns over the use of total body irradiation in systemic sclerosis. Bone Marrow Transplant 2004; 34: 745-751.

16. Oyama Y, Barr WG, Statkute L, Corbridge T, Burt RK. Autologous non-myeloablative hematopoietic stem cell transplantation in patients with systemic sclerosis. Bone Marrow Transplant. 2007; 40(6):549-55.

17. Vonk MC, Marjanovic Z, van den Hoogen FH, van Laar JM, Farge $\mathrm{D}$, et al. Long-term follow-up results after autologous haematopoietic stem cell transplantation for severe systemic sclerosis. Ann Rheum Dis. 2008;67(1):98-104.

18. Farge D, van Laar JM, Tyndall A. The European randomized HSCT trial for scleroderma. Blood Marrow Transplant. 2004;14: 7-9.

19. Farge D, Nash R, Laar JM. Autologous stem cell transplantation for systemic sclerosis. Autoimmunity. 2008;41(8):416-624

20. Miniati I, Conforti ML, Bernardo P, Tyndall A, Gensini GF, MatucciCerinic M. Hematopoietic stem cell transplantation in autoimmune diseases: algorithm for cardiovascular assessment. Herz. 2007; 32(1):43-50.

21. Morandi P, Ruffini PA, Benvenuto GM, Raimondi R, Fosser V. Cardiac toxicity of high-dose chemotherapy. Bone Marrow Transplant. 2005;35(4):323-34.

22. Komatsuda A, Kawabata Y, Horiuchi T, Motegi M, Ozawa M, Sawada K, et al. Successful autologous peripheral blood stem cell transplantation using thiotepa in a patient with systemic sclerosis and cardiac involvement. Tohoku J Exp Med. 2006;209(1):61-7.

23. Coghlan JG, Handler CE, Kottaridis PD. Cardiac assessment of patients for haematopoietic stem cell transplantation. Best Pract Res Clin Haematol. 2007;20(2):247-63.

24. Shiratsuchi M, Motomura S, Abe Y, Shiokawa S, Nishimura J. Longterm follow-up after nonmyeloablative allogeneic hematopoietic stem cell transplantation for systemic sclerosis. Clin Rheumatol. 2008; 27(9):1207-9.

25. Loh Y, Oyama Y, Statkute L, Verda L, Burt RK, et al. Nonmyeloablative allogeneic hematopoietic stem cell transplantation for severe systemic sclerosis: graft-versus-autoimmunity without graft-versus-host disease? Bone Marrow Transplant. 2007;39(7): 435-7.

26. Slaper-Cortenbach IC, Wijngaarden-du Bois MJ, de Vries-van Rossen A, Borst HP, van der Lelie H, van Heugten HG, et al. The depletion of $\mathrm{T}$ cells from haematopoietic stem cell transplants. Rheumatology (Oxford) 1999;38(8):751-4.

27. Webb IJ, Coral FS, Andersen JW, Elias AD, Finberg RW, Nadler LM, et al. Sources and sequelae of bacterial contamination of hematopoietic stem cell components: implications for the safety of hematotherapy and graft engineering. Transfusion. 1996; 36(9):782-8

28. Snowden JA, Passweg J, Moore JJ, et al. Autologous hemopoietic stem cell transplantation in severe rheumatoid arthritis: a report from the EBMT and ABMTR. J Rheumatol. 2004;31(3):482-8.

29. Moore J, Brooks P, Milliken S, Biggs J, Ma D, Handel M, et al. A pilot randomized trial comparing CD34-selected versus unmanipulated hemopoietic stem cell transplantation for severe refractory rheumatoid arthritis. Arthritis Rheum 2004;46(9): $2301-9$.

30. Czirják L, Foeldvari I, Müller-Ladner U. Skin involvement in systemic sclerosis. Rheumatology (Oxford). 2008;47(Suppl 5): 44-5.

31. Miniati I, Guiducci S, Conforti ML, Rogai V, Saccardi R, Tyndall A, et al. Autologous stem cell transplantation improves microcirculation in systemic sclerosis. Ann Rheum Dis. 2009;68(1):94-8.

32. Nevskaya T, Ananieva L, Bykovskaia S, Eremin I, Karandashov E, Nassonov E, et al. Autologous progenitor cell implantation as a novel therapeutic intervention for ischaemic digits in systemic sclerosis. Rheumatology. 2009;48(1):61-4.

33. Toledano C, Henegar C, Ilie D, Launay D, Tiev K, Farge D, et al. Cardiopulmonary function before and after cyclophosphamide treatment in severe systemic sclerosis: comparison of monthly intravenous bolus and autologous haematopoietic stem cell transplantation. Rev Med Interne. 2005;26(6):444-52.

34. Moraes D, Rodrigues MC, Stracieri ABPL, Barros G, Pieroni F, Cunha $\mathrm{C}$ et al. Autologous hematopoietic stem cell transplantation for systemic sclerosis. Rev Bras Hematol Hemoter. 2009:31(Supl 3)abstract 180:104.

35. Voltarelli JC, Stracieri ABPL, Oliveira, MCB, et al. Transplante de células-tronco hematopoéticas em doenças reumáticas- Parte 2: Experiência brasileira e perspectivas futuras. Rev Bras Reumatol $2005 ; 45: 301-12$

36. Fassas A, Anagnostopoulos A, Kazis A, et al. Peripheral blood stem cell transplantation in the treatment of progressive multiple sclerosis: First results of a pilot study. Bone Marrow Transplant 1997;20(8):631-8

37. Fassas A, Passweg JR, Anagnostopoulos A, Kazis A, Kozak T, Havrdova E, et al. Hematopoietic stem cell transplantation for multiple sclerosis. A retrospective multicenter study. J Neurol. 2002;249(8):1088-97.

38. Saccardi R, Kozak T, Bocelli-Tyndall C, Fassas A, Kazis A, Havrdova $\mathrm{E}$, et al. Autologous stem cell transplantation for progressive multiple sclerosis: update of the European Group for Blood and Marrow Transplantation autoimmune diseases working party database. Mult Scler. 2006;12(6):814-23.

39. Saccardi R, Mancardi GL, Solari A, Bosi A, Bruzzi P, Di Bartolomeo $\mathrm{P}$, et al. Autologous HSCT for severe progressive multiple sclerosis in a multicenter trial: impact on disease activity and quality of life. Blood. 2005;105(6):2601-7.

40. Saccardi R, Mancardi G, Bosi A, Bruzzi P, Di Bartolomeo P, Donelli A, et al. Autologous HSCT for severe progressive multiple sclerosis in the Italian prospective, multicentre GITMO-Neuro trial: long- 
term follow-up. Bone Marrow Transplant. 2008;41(Suppl 1):S17 (abstract)

41. Burt RK, Cohen BA, Russell E, Spero K, Joshi A, Oyama Y, et al Hematopoietic stem cell transplantation for progressive multiple sclerosis: failure of a total body irradiation-based conditioning regimen to prevent disease progression in patients with high disability scores. Blood. 2003;102(7):2373-8.

42. Carreras E, Saiz A, Marín P, Martínez C, Rovira M, Villamor N, et al. CD34+ selected autologous peripheral blood stem cell transplantation for multiple sclerosis: report of toxicity and treatment results at one year of follow-up in 15 patients. Haematologica. 2003;88(3):306-14.

43. Saiz A, Blanco Y, Berenguer J, Gómez-Choco M, Carreras E, Arbizu T, Graus F. Clinical outcome 6 years after autologous hematopoietic stem cell transplantation in multiple sclerosis. Neurologia. 2008; 23(7):405-7.

44. Nash RA, Bowen JD, McSweeney PA, Pavletic SZ, Maravilla KR, Park MS, et al. High-dose immunosuppressive therapy and autologous peripheral blood stem cell transplantation for severe multiple sclerosis. Blood. 2003;102(7):2364-72.

45. Kozák T, Havrdová E, Pit'ha J, Gregora E, Pytlík R, Maaloufová $\mathrm{J}$, et al. Immunoablative therapy with autologous stem cell transplantation in the treatment of poor risk multiple sclerosis. Transplant Proc. 2001;33(3):2179-81

46. Kozak T, Havrdova E, Pitha J, Mayerova K, Novakova L, Trneny $\mathrm{M}$, et al. Immunoablative therapy with autologous PBPC transplantation in the treatment of poor-risk multiple sclerosis. Bone Marrow Transplant. 2008; 41(Suppl 1): S18 (abstract).

47. Openshaw H, Lund BT, Kashyap A, Atkinson R, Sniecinski I, Weiner LP, et al. Peripheral blood stem cell transplantation in multiple sclerosis with busulfan and cyclophosphamide conditioning: report of toxicity and immunological monitoring. Biol Blood Marrow Transplant. 2000;6(5A):563-75.

48. Samijn JP, te Boekhorst PA, Mondria T, van Doorn PA, Flach HZ, van der Meché FG, et al. Intense $T$ cell depletion followed by autologous bone marrow transplantation for severe multiple sclerosis. J Neurol Neurosurg Psychiatry 2006;77(1):46-50.

49. Shevchenko YL, Novik AA, Kuznetsov AN, Afanasiev BV, Lisukov IA, Kozlov VA, et al. High-dose immunosuppressive therapy with autologous hematopoietic stem cell transplantation as a treatment option in multiple sclerosis. Exp Hematol 2008;36(8):922-8.

50. Freedman MS, Atkins HL, Arnold DL, Bar-Or A, on behalf of the Canadian BMT Study Group. Immune ablation and autologous stem cell transplantation for aggressive multiple sclerosis: interim 5-year report. Mult Scler. 2007;13 (Supp 2): S22 (abstract).
51. Su L, Xu J, Ji BX, Wan SG, Lu CY, Dong HQ et al. Autologous peripheral blood stem cell transplantation for severe multiple sclerosis. Int J Hematol 2006;84(3):276-81.

52. Xu J, Ji BX, Su L, Dong HQ, Sun XJ, Liu CY. Clinical outcomes after autologous haematopoietic stem cell transplantation in patients with progressive multiple sclerosis. Chin Med J (Engl) 2006;119(22):1851-18.

53. Ni XS, Ouyang J, Zhu WH, Wang C, Chen B. Autologous hematopoietic stem cell transplantation for progressive multiple sclerosis: report of efficacy and safety at three yr of follow up in 21 patients. Clin Transplant. 2006;20(4):485-9.

54. Fassas A, Mancardi GL. Autologous hemopoietic stem cell transplantation for multiple sclerosis: is it worthwile? Autoimmunity. 2009;41(8):601-10.

55. Hamerschlak N, Rodrigues M, Moraes DA, Oliveira MC, Stracieri ABPL, Pieroni $\mathrm{F}$, et al. Brazilian experience with two condidioting regimens in patients with multiple sclerosis: BEAM/horse ATG and CY/rabbit ATG. Bone Marrow Transplant. 2009; epub ahead of print.

Este artigo foi avaliado pelos coordenadores das Diretrizes do Transplante de Medula Óssea da Sociedade Brasileira de Transplante de Medula Óssea, Luis Fernando Bouzas, Prof. Julio Cesar Voltarelli e Nelson Hamerschlak, e publicado após avaliação e revisão do editor, Milton Artur Ruiz.

Conflito de interesse: sem conflito de interesse

Recebido: 28/10/2009

Aceito: 23/11/2009 